\title{
Untersuchungen über die Verbreitung der Heringslarven im Englischen Kanal und der südlichen Nordsee im Januar $1959^{1}$ )
}

\author{
Von Gotthilf Hempel \\ Aus der Biologischen Anstalt Helgoland \\ (Mit 1 Zahlentafel und 6 Abbildungen)
}

Hauptaufgabe der Forschungsfahrt von FFS „Anton Dohrn“ vom 5. bis 30. Januar war die großräumige Erfassung der Heringsbrut in der Nordsee südlich $55^{\circ} \mathrm{N}$ und im gesamten Englischen Kanal. Nach britischem und holländischem Vorbild wurde auf dieser Fahrt eine Planktonröhre nach GEHRINGER (1952) zum Fang der Heringslarven verwendet. Die vorliegende Mitteilung soll sich auf die Darstellung der Fangmethode und der mit ihr erzielten Ergebnisse beschränken. Eine Diskussion der hier gebotenen Verbreitungskarten und Rückschlüsse auf die Bedeutung der einzelnen Laichplätze, die Individualentwidklung und den Transport der Larven soll einer gemeinsamen Veröffentlichung der Ergebnisse der englischen, holländischen, schottischen und deutschen Forschungsfahrten des Winters $1958 / 59$ vorbehalten bleiben.

Durch diese Fahrten sollte die Verbreitung der verschiedenen im Spätherbst und Winter in der südlichen Nordsee und im Ostteil des Englischen Kanals schlüpfenden Larvenpopulationen über längere Zeit verfolgt werden. Die Untersuchungen waren außerdem im Hinblick auf die Frage nach den Veränderungen in den Heringsbeständen der südlichen Nordsee geplant. Es galt festzustellen, ob sich die Lage und die Bedeutung der Laichplätze im Untersuchungsgebiet gegenüber der Vorkriegszeit wesentlich geändert haben. Die Ergebnisse der Fahrten, die in jenen Jahren von den deutschen und englischen Forschungsschiffen „Poseidon", "George Bligh" und "Makrele" ausgeführt wurden, sind von BǘcKMANN 1951 zusammengefaßt worden. Ähnlich umfangreiche Untersuchungen über die Heringslarven in der südlichen Nordsee und im Kanal konnten bis 1958 nicht wieder durchgeführt werden Die deutschen Fahrten erfaßten die Situation jeweils nur in den Frühjahrsmonaten, audı blieben sie auf die südliche Nordsee oder einzelne ihrer Teilgebiete beschränkt:

"Gauss": März 1952, März 1953, März 1955; "Uthörn", ostfriesische Küste: April 1952 April 1953, März/April 1955; "Anton Dohrn“: März 1955, April 1956. Darstellung der Ergebnisse bei Bückmann 1954. Bohl 1954, BǘckmanN u. Hempel 1953, 1957.

Von englischen und holländischen Forschungsschiffen wurden in den letzten Jahren mehrfach kurze Forschungsfahrten in die Umgebung der Laich-

1) Herrn Prof. Dr. A. Bückmann zum 60. Geburtstag gewidmet. 
plätze des östlichen Kanals und in die südliche Flämische Bucht unternommen, die ganz oder teilweise der Erfassung der Heringsbrut dienten. Bisher sind nur einige Verbreitungskarten von diesen Fahrten publiziert worden (SImpson $1948,1949)$.

Der Hamburgische Forschungsrat, die Niedersächsische Landesregierung und die Deutsche Wissenschaftliche Kommission für Meeresforschung haben durch die Bereitstellung von Sachmitteln den Bau mehrerer Planktonröhren, die Anschaffung von Scherfüßen und anderen Hilfsmitteln ermöglicht. Das Deutsche Hydrographische Institut lieh uns einen Tiefenschreiber. $\mathrm{Z}_{\mathrm{u}}$ großem Dank sind wir ferner Herm J. J. Zijlstra und dem Rijksinstituut voor Visserij Onderzoek, Ijmuiden für die Oberlassung von Konstruktionszeichnungen und vielfache Beratung verpllichtet. Auch durften wir zeitweise einen Strommesser des genannten Instituts in unsere Planktonröhre einbauen. Dr. G. Glover, Scottish Marine Biological Association, Edinburgh, vermittelte uns den Ankauf der Scherfüße.

\section{Verlauf der Fahrt und hydrographische Situation}

Aus den hydrograph. Karten Abb. 1 u. 2 ist das Stationsnetz der Fahrt zu ersehen. Die Fahrt gliedert sich in zwei Abschnitte: Die Hauptgebiete des Larvenvorkommens in der Flämischen Bucht und im östlichen Kanal wurden zweimal erfaßt, am 13.-16. 1. und am 26.-28. 1. 1959. Während dieser Tage begünstigte ruhiges Wetter die Untersuchungen. Es wurde oftmals nicht nach einem starren Stationsprogramm gearbeitet, sondern durch Stichfahrten und zusätzliche Stationen versucht, die Grenzen der einzelnen Larvenvorkommen möglichst klar zu umreißen. Zwei N-S-Schnitte im Englischen Kanal bei $2^{0} \mathrm{~W}$ und $5^{\circ} \mathrm{W}$, sowie eine Reihe von Fängen unter der englischen Küste, an der Küste der Normandie und in der Keltischen See sollten u. a. der Feststellung dienen, ob in diesen z. T. fischereibiologisch weniger gut untersuchten Gebieten Heringslarven in nennenswerter Menge vorkommen. Diese Suche war praktisch vergeblich. Die Masse der an der Südküste Irlands abgelegten Heringsbrut scheint am 24. Januar noch nicht geschlüpft gewesen zu sein.

Die hydrographische Situation wurde durch ein enges Beobachtungsnetz erfaßt. Wie im Winter nicht anders zu erwarten, herrschte außerhalb der Küstengebiete Homogenität bezüglich der vertikalen Temperatur- und Salzgehaltsverteilung. Es genügt daher, hier die von Dr. Rogalda gezeichneten Karten der Verteilung von Temperatur und Salzgehalt am Boden wiederzugeben (Abb. 1 u. 2). In der Flämischen Bucht fällt die weite Ausdehnung des Küstenwassers vor den Küsten Belgiens und Hollands, sowie der abgeschnürte Kern salzreichen, warmen Kanalwassers auf. Ein solches vom Wind bestimmtes, schubartiges Vordringen atlantischer Wassermassen durch die Straße von Dover ist u. a. auch auf den Frühjahrs-Fahrten der "Gauss" beobachtet worden.

\section{Methode}

Quantitative Proben von Heringslarven verschiedenen Alters zu gewinnen, bereitet erhebliche Schwierigkeiten. In den letzten Jahren hat sich dafür die Verwendung des von GeHringer (1952) entwickelten Gulf III-Sampler auch in Europa durchgesetzt, der vom fahrenden Schiff aus mit Hilfe eines Scherkörpers in die gewünschte Wassertiefe gebracht wird. Eingehende Untersuchungen über die Fangeigenschaften dieses Gerätes hat BRIDGER (1956, 1958) angestellt. Das Gerät erfaßt die älteren, aktiveren Heringslarven besser als 


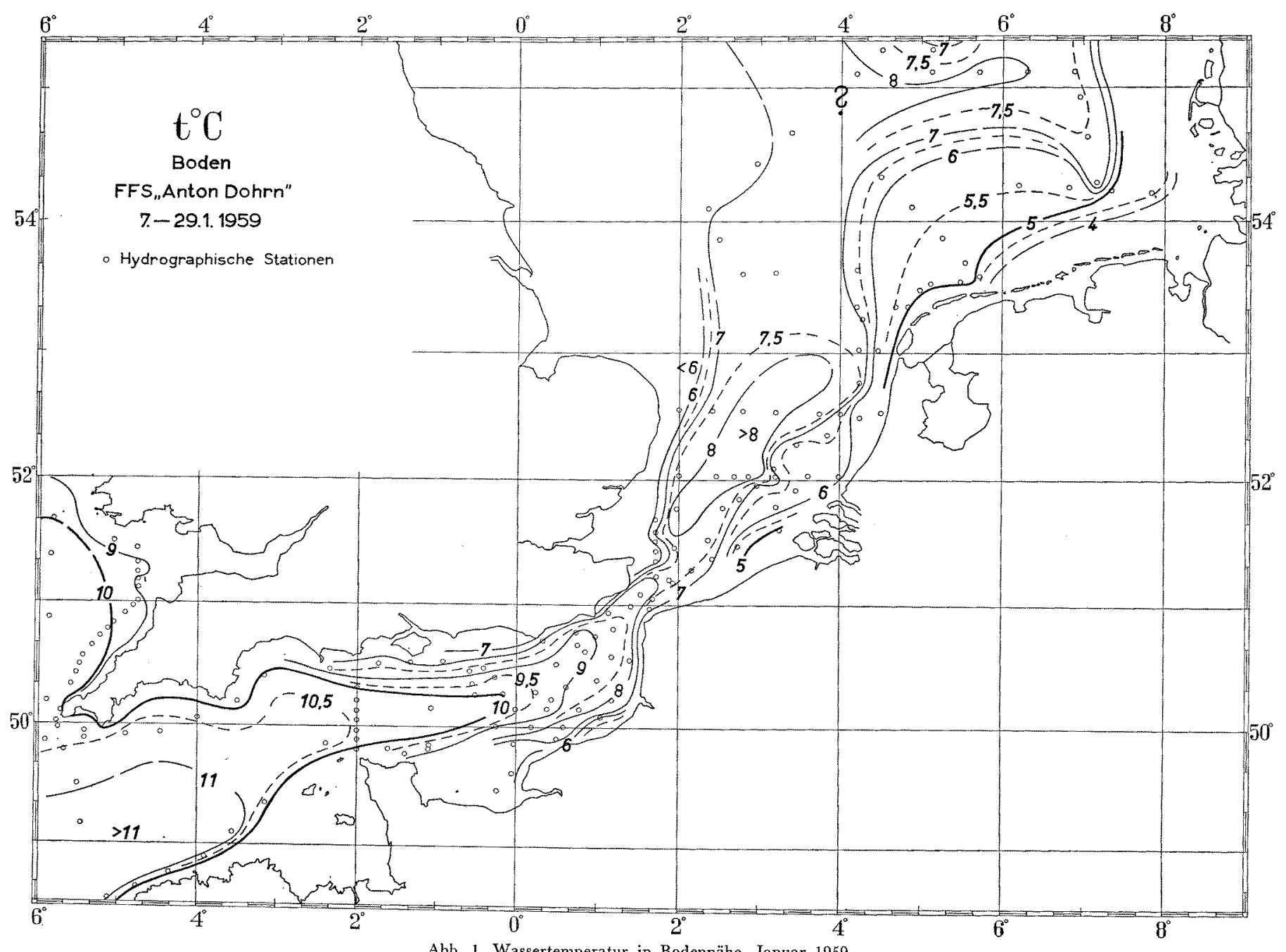

Abb. 1. Wassertemperatur in Bodennähe, Januar 1959

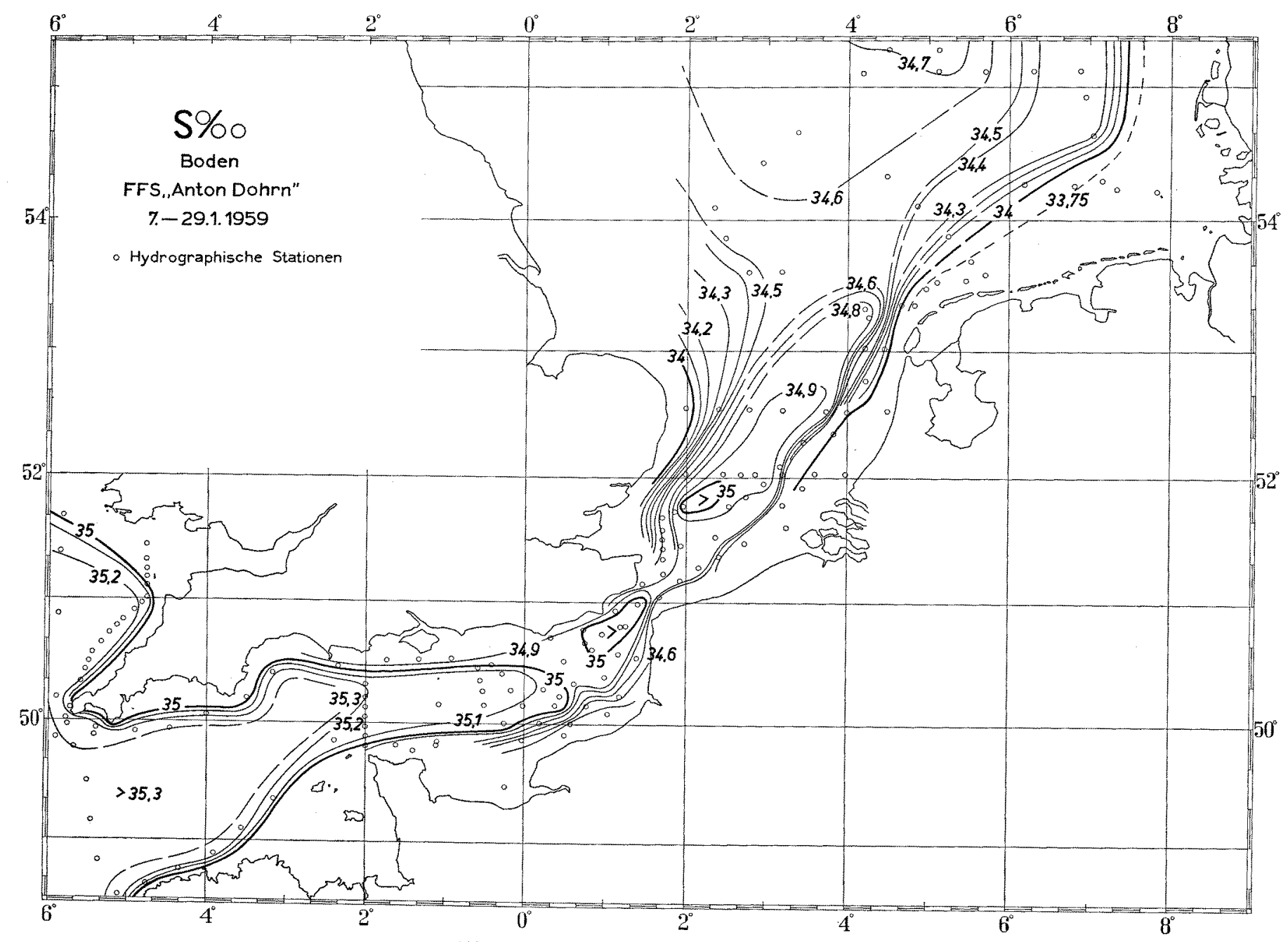


die bisher verwendeten Netze (Helgoländer Larvennetz und Petersen's Jungfischtrawl).

Im praktischen Gebrauch hat sich der Gulf III-Sampler sehr gut bewährt. Im Vergleich zu den vertikal fischenden Planktonnetzen spart sein Einsatz Zeit und ist auch bei schlechtem Wetter möglich. So kann bis Windstärke $9 \mathrm{Bft}$

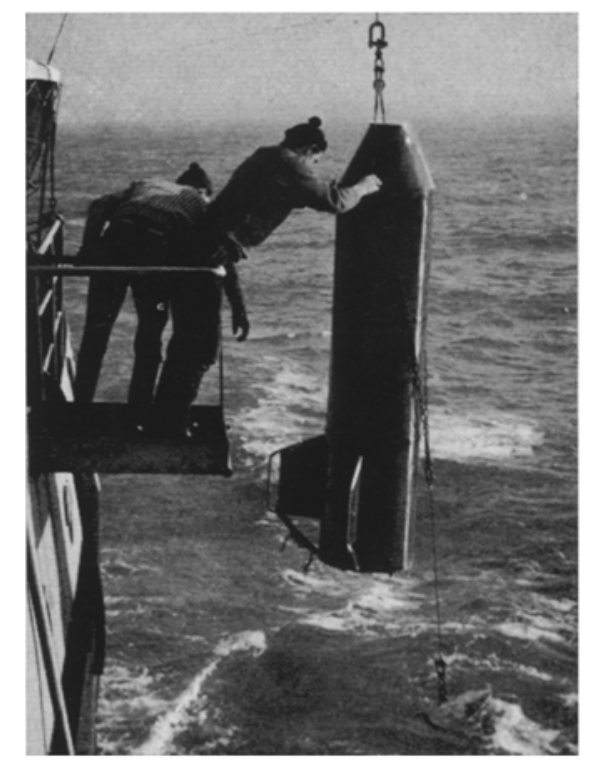

Abb. 3. Der "Hai“ mit kalifornischem Scherfuß. In dem vorn zugespitzten Hohlzylinder befindet sich ein herausnehmbares Leichtmetallgestänge, in dem das $1,4 \mathrm{~m}$ lange, konische Netz mit Becher zum Sammeln des Fanges befestigt ist. Hinter dem Netz ist der Strommesser angebracht.

mit der Planktonröhre gearbeitet werden. Bis Windstärke 6 wird beim Fang der jeweils anliegende Schiffskurs beibehalten. Nur bei stärkerem Seegang muß das Schiff vor dem Wind fahren, um die ruckweise Beanspruchung der Schlepptrosse zu mildern.

Die von uns verwendeten und als „Hai“ bezeichneten Modifikationen des Gulf III-Sampler (Abb. 3) weichen nur in wenigen, für die Fängigkeit unwesentlichen Einzelheiten von dem holländischen Gerät ab, dessen Konstruktionszeichnungen ihrem Bau zugrunde lagen:

Beim „Hai“ faßt die Aufhängung an beiden Seiten des Gerätes anstatt oben in der Mitte, und auch der Scherfuß, der nach dem kalifornischen Vorbild (AhLstrom et al., 1958 Fig.14) angefertigt wurde, hängt an zwei Trossen statt an einer. Die Halterung des Netzes im Schleppkörper ist beim "Hai" primitiver als bei dem holländischen Gerät.' Der Schleppkörper des "Hai" besteht aus verzinktem und gestrichenem Eisenblech, er ist dadurch erheblich schwerer als die Aluminiummodelle. Das Netzgestänge ist aus Aluminium, die Netzgaze aus Phosphorbronze') gefertigt. Bezüglich der Maschenweite (15,5 Maschen pro Zentimeter) und der Eintrittsöffnung für das zu filtrierende Wasser $(\phi 18-19 \mathrm{~cm})$ besteht Übereinstimmung mit dem holländischen Gerät.

Für die Fänge in der Flämischen Bucht und im Englischen Kanal konnten wir einen holländischen Strommesser verwenden, mit dessen Hilfe Vergleichs-

2) Beim längeren Gebraudh des „Hai“ erwies sich die Phosphorbronze als wenig geeignet. Durch Grünspan und darauf haftendes Plankton setzt sich die Gaze schnell dicht, was bei den aus Monelgaze gefertigten Netzen nicht auftreten soll. 
werte über die Menge des bei jedem Hol durchfischten Wassers gewonnen wurden.

Um Vergleiche mit den früheren Vertikalfängen durchführen zu können, muß der "Hai“ alle Wasserschichten möglichst gleichmäßig durchfischen. In Versuchen ergab sich, daß dies unter den technischen Gegebenheiten von FFS „Anton Dohrn" am besten gewährleistet ist, wenn das Gerät bei konstanter Fahrt des Schiffes möglichst langsam gefiert und unmittelbar darauf wieder langsam gehievt wird, sobald man das Gerät in der gewünschten Tiefe, d. h. meist in Bodennähe vermutet. Die durchschnittliche minimale Fiergeschwindigkeit beträgt bei der verwendeten Winde etwa $0,4 \mathrm{~m} / \mathrm{sec}$, die Hievgeschwindigkeit ca. $0,25 \mathrm{~m} / \mathrm{sec}$. In den meisten Fällen wurde bei 5,9-6,1 kn Fahrt des Schiffes gefischt, nur einige Fänge wurden wegen grober See bei 4 oder $5 \mathrm{kn}$ Fahrt gemacht. Die Fahrtstufe des Schiffes war jeweils beim Hieven und Fieren des Gerätes die gleiche, die Fahrt des „Hai“ durchs Wasser betrug demnach beim Fieren etwa $5 \mathrm{kn}$, beim Hieven dagegen $6^{1 / 2} \mathrm{kn}$. Versuche mit einem Tiefenschreiber ergaben, daß bei diesem Verfahren weder die oberen noch die tieferen Wasserschichten bevorzugt befischt werden, einheitliche Stromverhältnisse in allen Wasserschichten vorausgesetzt. In folgender Tabelle sind die Zeiten für die einzelnen 5 -m-Tiefenstufen von $0-35 \mathrm{~m}$ aus einem solchen Versuch aufgetragen, bei dem die mittlere Fiergeschwindigkeit $0,43 \mathrm{~m} / \mathrm{sec}$, die Hievgeschwindigkeit $0,24 \mathrm{~m} / \mathrm{sec}$ betrug.

Verweildauer (Minuten) des "Hai ${ }^{*}$ in den einzelnen Tiefenstufen (Abb.4)

\begin{tabular}{cccc}
\hline Tiefenstufe & Fieren & Hieven & Gesamt \\
\hline $0-5 \mathrm{~m}$ & 1,4 & 1,5 & 2,9 \\
$5-10$ & 1,5 & 1,7 & 3,2 \\
$10-15$ & 1,1 & 1,6 & 2,7 \\
$15-20$ & 1,3 & 1,9 & 3,2 \\
$20-25$ & 0,9 & 1,5 & 2,4 \\
$25-30$ & 1,2 & 2,1 & 3,3 \\
$30-35$ & 1,0 & 2,0 & 3,0
\end{tabular}

Einige Unsiderheit bestand bei der Abschätzung der erreichten Schlepptiefe, die aus dem Drahtwinkel und der jeweils ausgesteckten Trosse ( $\varnothing 8 \mathrm{~mm}$ ) errechnet wurde. Die Messung des Drahtwinkels war besonders bei unruhiger See, bei Nacht und wenn der "Hai" nicht genau achteraus stand, ungenau. Um eine Bodenberührung von Scherfuß und „Hai" zu vermeiden, wurde in solchen Fällen weniger Trosse gesteckt, als auf Grund des Drahtwinkels erforderlich gewesen wäre, um den "Hai" in Bodennähe zu bringen. Die bodennahen Wasserschichten wurden daher wahrscheinlich in vielen Fällen nicht erreicht. Eine Zusammenstellung der für die einzelnen Stationen errechneten Schlepptiefen ist in der Tabelle der Fangergebnisse (Zahlentafel 1) enthalten. Spätere Versuche zeigten, daß zumindest in den oberen $35 \mathrm{~m}$ (Meßbereich des verwendeten Tiefenschreibers) die aus dem Drahtwinkel berechneten Schlepptiefen recht gut mit den vom Tiefenschreiber registrierten übereinstimmten (Abb. 4). Lediglich beim Hieven steht die Planktonröhre anscheinend etwas tiefer, als sich aus dem Drahtwinkel errechnen läßt. Diese Versuche wurden allerdings bei ruhigem Wetter und unter günstigen Meßbedingungen durchgeführt ${ }^{3}$ ).

3) Anm. b. d. Korr.: Neuerdings verwenden wir bei jedem Fang einen Tiefenschreiber (Meßbereich 0-70 m). 


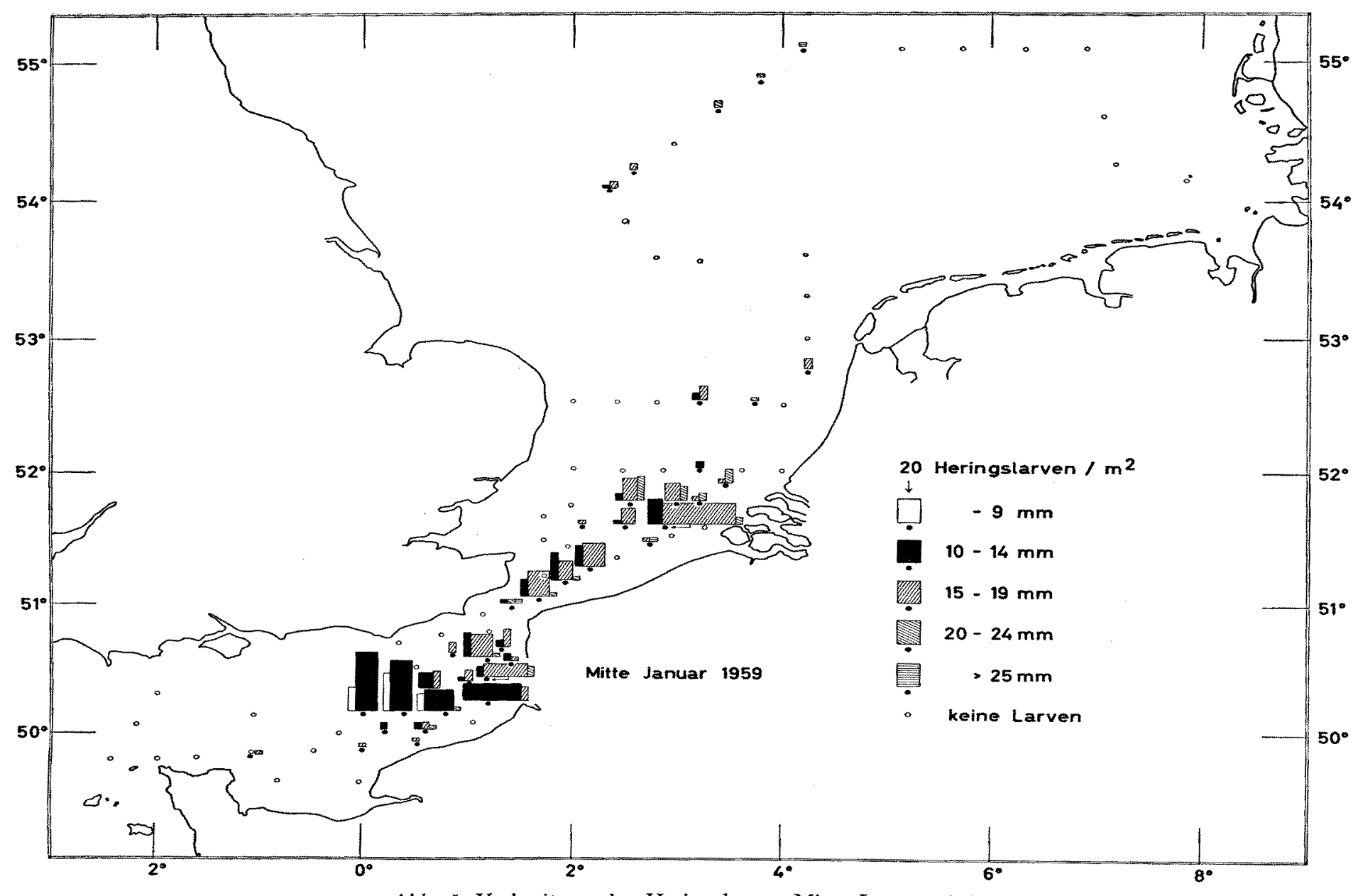

Abb. 5. Verbreitung der Heringslarven Mitte Januar 1959

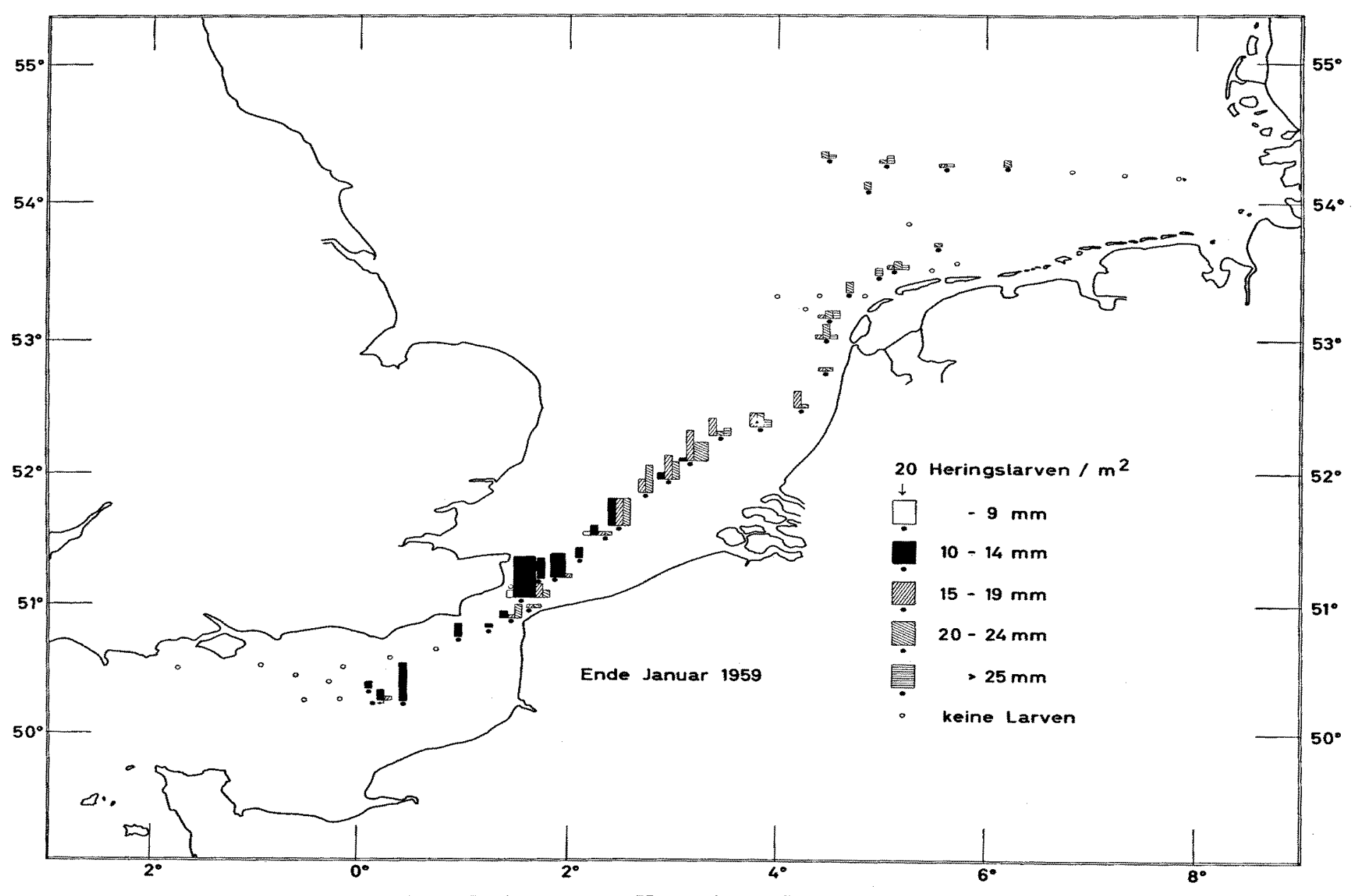

Abb. 6. Verbreitung der Heringslarven Ende Januar 1959 
In Zahlentafel 1 sind die Fangergebnisse als Stückzahl der Heringslarven in den einzelnen Millimetergruppen angegeben. Um diese Werte in Fang $/ \mathrm{m}^{2}$ umrechnen zu können, mußte für jeden Fang die Menge des filtrierten Wassers bekannt sein. Die Schleppstrecke wurde aus der Fahrtgeschwindigkeit und der

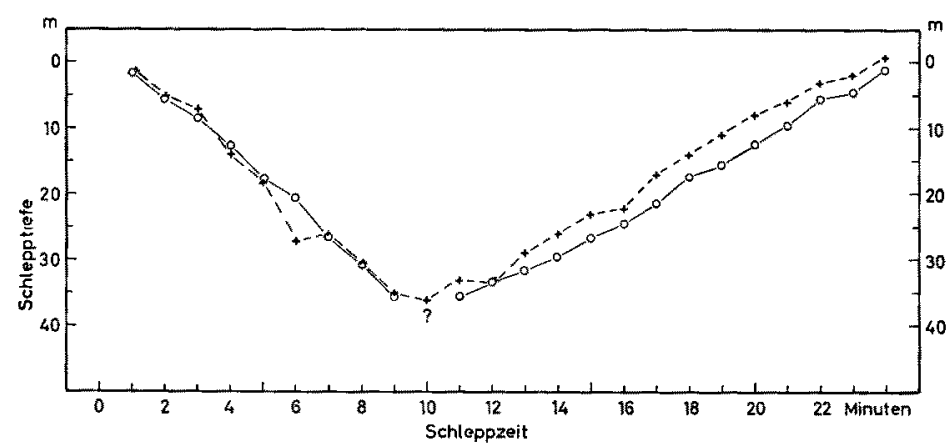

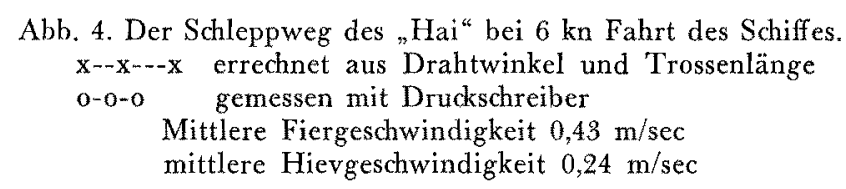

Schleppdauer vom Eintauchen des anfangs auf der Wasseroberfläche schwimmenden "Hai" bis zu seinem Wiederauftauchen berechnet und zum Vergleich das Log des Schiffes, das die zurückgelegte Distanz auf 1/10 sm genau angibt, notiert. Beide Werte stimmten meist sehr gut überein. Aus Bridgers Versuchen (1958) ergibt sich, daß der Filtrationswiderstand des Netzes die Menge des bei 5,8 kn Fahrt durch die Planktonröhre fließenden Wassers nicht beeinflußt, wenn die Einstromöffnung der Röhre auf etwa $20 \mathrm{~cm}$ Durchmesser bei $50 \mathrm{~cm}$ Röhrenlumen verjüngt ist. Wir können daher annehmen, daß vor der $18 \mathrm{~cm}$ weiten Offnung des "Hai" weder ein nennenswerter Stau, noch ein starker Sog auftreten, wenn das Netz mit $6 \mathrm{kn}$ Fahrt geschleppt wird. Die durchfischte Wassermasse hat demnach einen Querschnitt von etwa $0,025 \mathrm{~m}^{2}$, auf $1 \mathrm{sm}$ Schleppstrecke werden $47,0 \mathrm{~m}^{3}$ Wasser durchfischt. Entsprechend wurde für jede Station die durchfischte Wassermasse berechnet und diese Zahl zur Wassertiefe, d. h. der Wassermasse unter $1 \mathrm{~m}^{2}$ Oberfläche in Beziehung gesetzt. So ergab sich der Faktor für die Umrechnung des Gesamtfanges auf die Bestandsdichte unter $1 \mathrm{~m}^{2}$ Wasseroberfläche. Bei dem von uns angewendeten Verfahren der kontinuierlichen Schräghols lagen die Umrechnungsfaktoren meist zwischen 0,5 und 1 . Im Gegensatz zu Verfahren, die direkt die durch das Gerät strömende Wassermenge messen, führt die hier beschriebene Berechnungsmethode zu falschen Ergebnissen, wenn sich der Filtrationswiderstand des Netzes durch Verkleben der Maschen stark erhöht oder wenn in tieferen Wasserschichten abweichende Stromverhältnisse herrschen.

Eine bessere Möglichkeit zur Bestimmung der durchfischten Wassermenge bietet daher ein Strommesser. Nach den von $Z_{\text {IJLSTRA (briefliche Mitteilung) }}$ aufgestellten und neuerdings revidierten Eichwerten entspricht 1 Umdrehung des in den "Hai" eingebauten Strommessers etwa $0,17 \mathrm{~m}^{3}$ durchfischten Wassers. Für $1 \mathrm{sm}$ (nach Schiffslog) errechneten sich als Mittel von 102 Fängen 298,5 Umdrehungen, das entspricht nach der angegebenen Eichung 48,6 $\mathrm{m}^{3}$ durchfischten Wassers. Die aus dem Offnungsquerschnitt und aus den Zähl- 
werksangaben unabhängig berechneten Wassermengen stimmen erstaunlich gut überein. Dementsprechend sind auch die Umrechnungsfaktoren nach beiden Rechenwegen im Mittel annähernd gleich.

In denjenigen Fällen, in denen das Bodenwasser vom "Hai" nicht erfaßt wurde (s. o.), birgt die Umrechnung des Fanges auf die Wassersäule unter $1 \mathrm{~m}^{2}$ eine große Unsicherheit, da nicht bekannt ist, ob die bodennahen Wasserschichten die gleiche Besiedlungsdichte aufweisen wie die befischten Schichten. Bei Fischbrutuntersuchungen über großen Meerestiefen bietet sich die Umrechnung nach der Schlepptiefe an, die meist der angenommenen unteren Verbreitungsgrenze der Fischbrut $(50-100 \mathrm{~m})$ entsprechend gewählt wird. Die Umrechnung auf die Wassertiefe würde hier irreführende Umrechnungsfaktoren ergeben.

\section{Ergebnisse}

Die Fangergebnisse, umgerechnet auf die Anzahl der Heringslarven unter $1 \mathrm{~m}^{2}$ Oberfläche, sind für die 1. und 2. Teilfahrt getrennt in Abb. 5 und 6 dargestellt. Dazu wurde das Material nach 5-cm-Längengruppen eingeteilt. Die Maße beziehen sich auf mit Formalin konserviertes Material, die Larven sind also etwas geschrumpft. Gemessen wurde die Totallänge von der Schnauze bis zur Schwanzspitze. Um einen Vergleich mit den neueren ausländischen Veröffentlichungen zu ermöglichen, ist in Zahlentafel 1 auch eine Einteilung nach Entwicklungsstadien, wie sie BrIDgER (1956) anhand der Resorption des Dottersackes und der Ausbildung der Flossen beschrieben hat, wiedergegeben. Anhand ihrer Verbreitung und ihrer Körperlänge konnten insgesamt 5 Gruppen von Heringslarven im Untersuchungsgebiet unterschieden werden.

1. Isoliert von den Larven der Flämischen Bucht wurden auf der ersten und zweiten Kreuzfahrt nördlich $54^{\circ} \mathrm{N}$ einzelne, meist große Larven von 16 bis $33 \mathrm{~mm}$ Körperlänge gefangen. Die Zahl der Larven pro $\mathrm{m}^{2}$ betrug nur 2 bis 3. Die Heringslarven hatten die Deutsche Bucht im Januar offenbar noch nicht erreicht.

2. Die nördlichen Vorläufer der großen Larvenpopulation in der Flämischen Bucht standen Mitte Januar im Kanalwasser querab Ijmuiden. Wie bei der ganzen Population herrschten hier Tiere von 15-19 mm Totallänge vor. Fänge am Ostrand des salzreichen, warmen Wassers waren um diese Zeit noch vergeblich. Die Hauptmasse der Larven, bis ca. 70 Tiere $/ \mathrm{m}^{2}$ stand vor Seeland außerhalb des Küstenwassers (mittlere Länge 17,1 mm). Der Westteil der Flämischen Bucht war ganz frei von Heringslarven. 14 Tage später waren die Larven ins holländische Küstenwasser bei Ijmuiden eingedrungen und im Küstenstrom weit nach Norden verfrachtet worden. Durch Stichfahrten konnte festgestellt werden, daß vor Texel die Verbreitung der Larven auf das Küstenwasser beschränkt war und daß am 28. 1. die ersten Larven die Gewässer vor Terschelling erreicht hatten. Während die vor Seeland konzentrierten Larven am 26. Januar im Mittel etwa $19 \mathrm{~mm}$ lang waren, maßen die im Küstenwasser gefangenen Vorläufer fast ausschließlich über $20 \mathrm{~mm}$. Die mittlere länge beim Gros der Larven, das sich nach Nordosten ausgebreitet hatte, lag nun bei $19,8 \mathrm{~mm}$.

3. Im nordöstlichen Kanalausgang wurde eine dritte Gruppe von Larven beobachtet, deren Bestandsdichte etwa 30 Tiere/ $\mathrm{m}^{2}$ betrug. Die mittlere Länge lag Mitte Januar bei $15,9 \mathrm{~mm}$, d. h. nur wenig niedriger als bei der vorgenannten Gruppe. Ende Januar standen diese Larven anscheinend vor Seeland und batten Anschluß an die nördlichere Gruppe (Gr. 2) gefunden. 

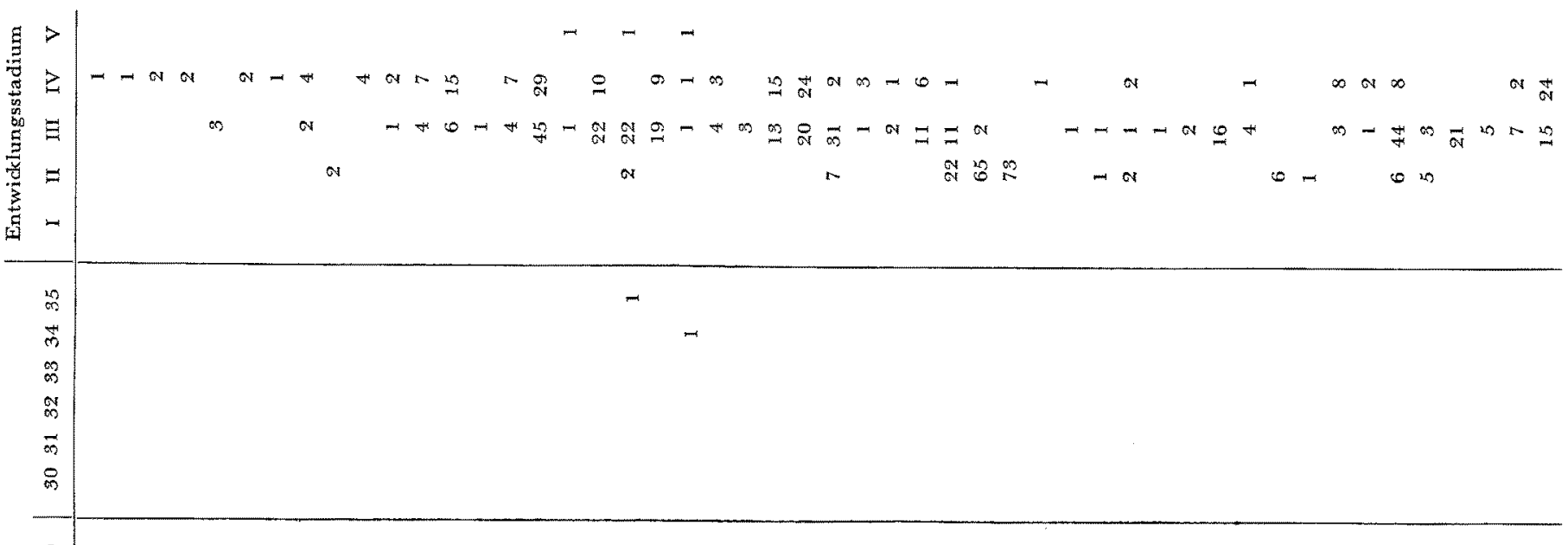

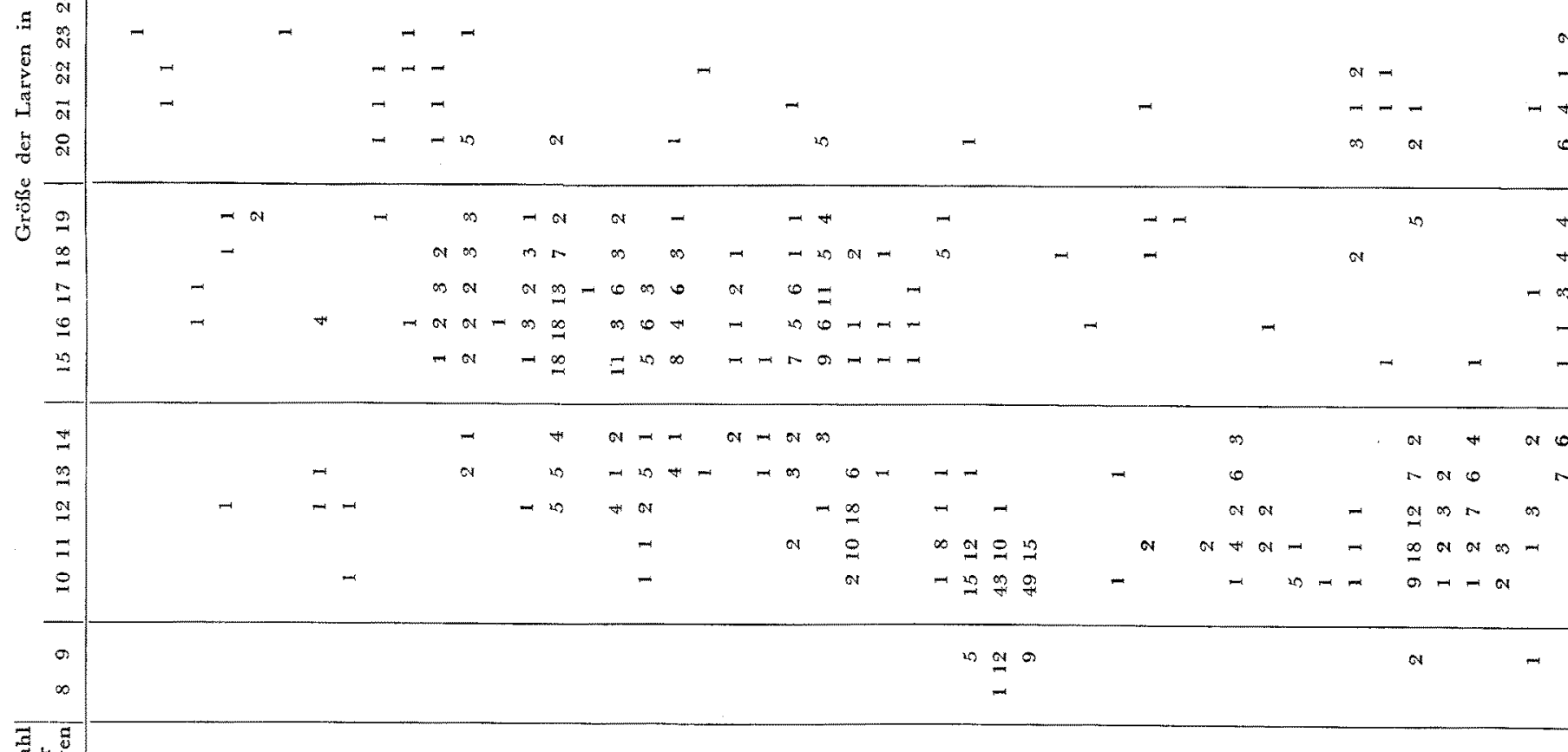

\section{.}

\begin{tabular}{|c|c|c|}
\hline 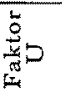 & 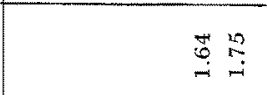 & 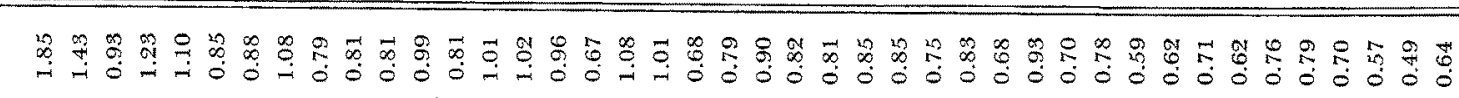 \\
\hline
\end{tabular}

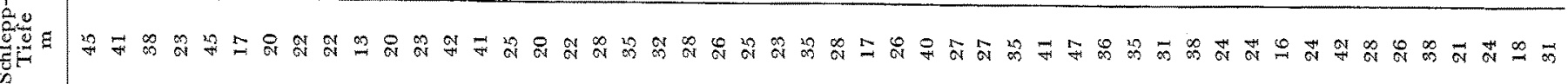

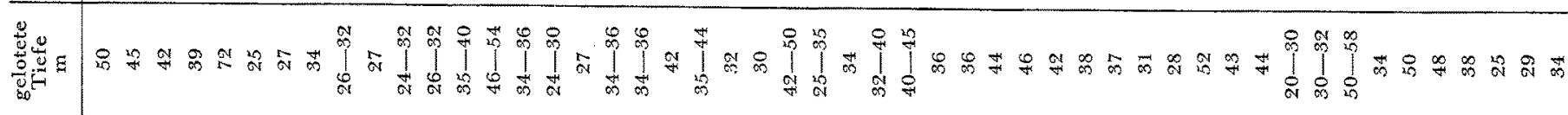

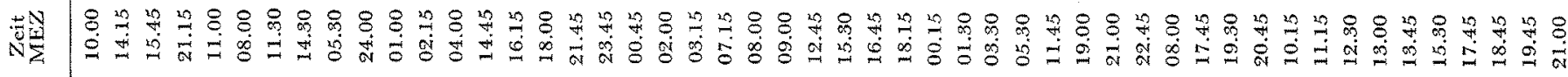

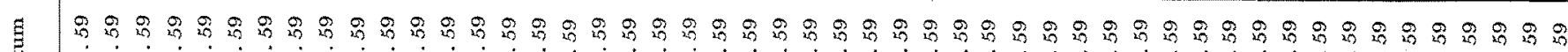
跑

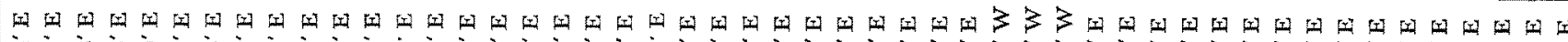

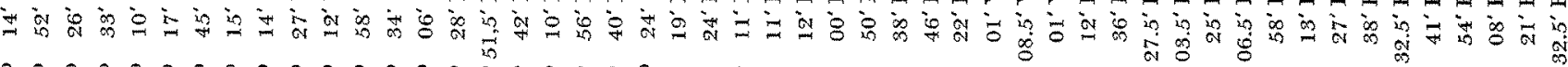

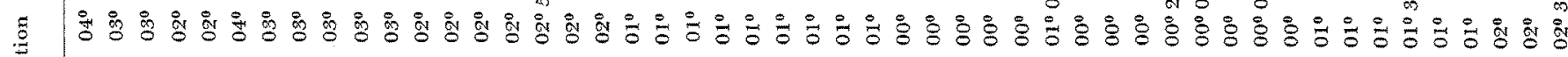

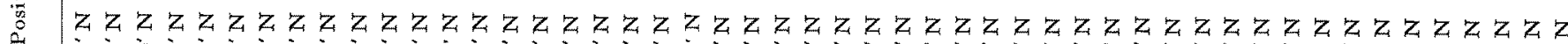

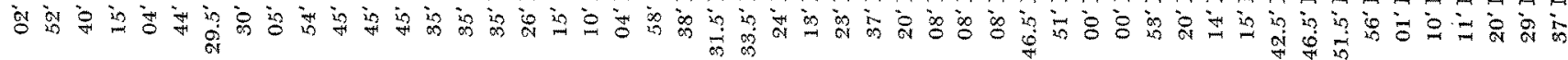

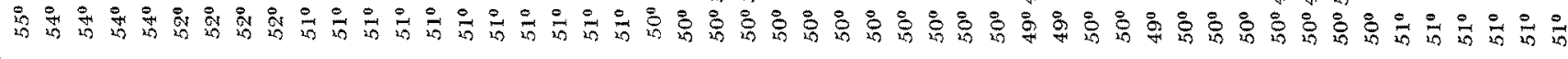


Die Heringslarven, die in großer Zahl (bis 70 Tiere $/ \mathrm{m}^{2}$ ) im östlichen Kanal gefangen wurden, scheinen zwei Laichakten zu entstammen:

4. Die nördlich $50^{\circ} 20^{\prime} \mathrm{N}$ gefangenen Larven waren meist $>15 \mathrm{~mm}$ $(\bar{x}=16,6 \mathrm{~mm})$. Auf dem 2. Teil der Reise wurden diese Larven nicht als selbständige Gruppe beobachtet. Vielleicht standen sie um diese Zeit nahe der Küste bei Calais, einige mögen auch der folgenden Gruppe beigemischt gewesen sein.

5. Die Mitte Januar südlich und westlich dieser Gruppe angetroffenen Heringslarven waren bedeutend kleiner $(\overrightarrow{\mathrm{x}}=11,5 \mathrm{~mm})$, Dottersacklarven wurden jedoch auch hier nicht gefangen. Die Verbreitung dieser jungen Larven war Ende Januar wenig einheitlich, einige von ihnen standen im östlichen Kanal, eine reichere Ansammlung in der Straße von Dover, dort betrug die Durchschnittslänge der Larven $14,3 \mathrm{~mm}$. Die Verbreitung der Heringslarven hatte ihre scharfe Westgrenze am 0-Meridian, die englische Seite des Kanals war auch östlich davon frei von Heringslarven.

Die Wiederholung der Aufnahme nach etwa 14 Tagen durch eine zweite Kreuzfahrt erlaubte nicht nur Rückschlüsse auf den Transport der Heringslarven, sondern lieferte auch einige Anhaltspunkte über das Wachstum der Larven bei $7-9^{\circ} \mathrm{C}$. Für die Hauptmasse der Larvengruppe 2 errechnet sich ein Zuwachs von $2,7 \mathrm{~mm}$, für die Larvengruppe 5 ein solcher von $2,8 \mathrm{~mm}$. Diese Zuwachsraten stimmen mit den von BüCKMANN 1951 beschriebenen überein, der einen monatlichen Zuwachs von $2-6 \mathrm{~mm}$ feststellte. Allerdings ist nicht ausgeschlossen, daß Ende Januar den genannten Gruppen Tiere der Gruppen 3 bzw. 5 beigemischt waren. Das würde bedeuten, daß wir bei Larvengruppe 2 den Zuwachs unterschätzen, bei Gruppe 5 aber überschätzen.

Wie eingangs hervorgehoben, erhalten die Ergebnisse der Januar-Reise über die Durchschnittslängen und die Verbreitungsgebiete ihre volle Bedeutung erst, wenn sie mit den Befunden der anderen Forschungsfahrten, auf welchen die Situation während und unmittelbar nach der Laichzeit erfaßt wurde, kombiniert werden können.

\section{Zusammenfassung}

Auf 177 Stationen, die über die Nordsee südlich $55^{\circ} \mathrm{N}$ und den Englischen Kanal verteilt waren, wurde im Januar 1959 das Vorkommen von Heringslarven untersucht. Die Fänge wurden mit einem als „Hai“ bezeichneten, in einigen Einzelheiten abgewandelten Gulf III-Sampler nach GeHringer durchgeführt.

Unter langsamen, kontinuierlichen Fieren und Hieven des Gerätes $(0,4$ bzw. $0,25 \mathrm{~m} / \mathrm{sec}$ bei $6 \mathrm{kn}$ Fahrt) konnten bei Schräghols alle Wasserschichten hinreichend gleichmäßig durchfischt werden. Bei günstigen Ablesebedingungen liefert die Angabe von Drahtwinkel und Trossenlänge im flachen Wasser der südlichen Nordsee brauchbare Werte für die Schlepptiefe.

Die Menge des filtrierten Wassers wurde sowohl aus dem Offnungsquerschnitt des "Hai" als auch aus Strommesserangaben berechnet. Im Mittel stimmen beide Werte gut überein. Sie bilden die Grundlage für die Berechnung der Larvenzahl unter $1 \mathrm{~m}^{2}$ Meeresoberfläche

Die Verbreitung der Heringslarven Mitte und Ende Januar 1959 ist in den Karten Abb. 5 und 6 wiedergegeben. Das Auftreten von 5 räumlich von- 
einander getrennten und durch die Größenverteilung unterschiedenen Larvengruppen wird kurz beschrieben.

Eine Diskussion der Resultate im Hinblick auf die Herkunft und Volksstärke der einzelnen Larvengruppen, auf ihre Verfrachtung, Ausbreitung und Verlustrate sowie auf das Individualwachstum soll in Verbindung mit der Darstellung der Ergebnisse britischer und holländischer Forschungsfahrten demnächst andernorts gegeben werden.

\section{Summary}

The distribution of the herring larvae in the southern North Sea and the English Channel was investigated in January 1959 during a cruise of RV „Anton Dohrn“.

The samples were taken by oblique hauls of a modified Gulf III-Sampler (,Hai"). The technique of handling the gear and of calculating the number of larvae per $\mathrm{m}^{2}$ is described in full. Tests by means of a depth recorder showed two results: 1 . Up to 20 fathoms at least all strata have been fished equally by a continuous slow shooting and hauling the sampler. 2. The measurements of the wear angle can fairly be used for calculating the depth of fishing.

The distribution of herring larvae according to their length and their stage of development is shown in table 1 and in two maps. A discussion will be given later in connection with the results of Dutch and British cruises in November 1958 to February 1959.

\section{Literaturverzeichnis}

1. Ahlstrom, E. H., J. D. Isaacs, J. R. Thrailkill, L. W. Kidd, 1958: High-speed plankton sampler. U.S. Dept. Int., Fish Wildl. Scrv. Fish. Bull. 132.

2. Boh 1, H. J., 1954: (Herring): Larvae in the Southern North Sea and the German Bight during March 1953. Cons. perm. Intetn. Explor. Mer, Ann. Biol. 10, 151-152.

3. Bridger, J. P., 1956: On Day and Night Variation in Catches of Fish Larvac. J. Cons. perm. Intern. Explor. Mer 22, 42-57.

4. Bridger, J. P., 1958: On efficiency tests made with a modified Gulf III High-Speed Tow-Net. J. Cons. Intern. Explor. Mer 23, 357-365.

5. B ückmann, A., 1951: Die Untersuchungen der Biologischen Anstalt über die Okologie der Heringsbrut in der südlichen Nordsee. I. Helgol. Wiss. Meeresunters. 3, 1-57.

6. Bückmann, A., 1954: Fischbrut und Evertebraten-Großplankton auf der Fahrt der "Gauss" im Februar/März 1952. Ber. Dtsch. Wiss. Komm. Meeresforsch. 13, 154-173.

7. Bückmann, A. u. G. Hempel, 1953: Beobachtungen über Verteilung und Entwicklungszustand der Heringslarven an der west- und ostfriesischen Küste im Frühjahr 1952. Kurze Mitteil. fischereibiolog. Abt. d. MPI f. Meeresbiol. Wilhelmshaven 2, 24-38.

8. Bückmann, A. u. G. Hempe1, 1957: Untersuchungen an der Heringslarvenbevölkerung der Innenjade. Helgol. Wiss. Meeresunters. 6, 52-70.

9. Gehringer, J. W., 1952: High speed Plankton samplers. 2. An all-metal plankton sampler (Model Gulf III). U.S. Dept. Int., Fish. Wildl. Serv., Spec, Sci. Rep. - Fish 88.

10. Rogalla, E.: Hydrographic conditions in the English Channel and the southern North Sea in January 1959. Cons. Intern. Explor. Mer, Ann. Biol. 16 (im Drudk).

11. S impson, A. C., 1949: Notes on the Occurrence of fish eggs and larvae in the Southern Bight of the North Sea during the Winter of 1946-47. Cons. Intern. Explor. Mer, Ann. Biol. 4, 90-95.

12. Simps on, A, C., 1949: Notes on the Occurrence of fish eggs and larvae in the Southern Bight of the North Sea during the Winter of 1947-48. Cons. Intern. Explor. Mer. Ann. Biol. 5, 90-97. 